\title{
Towards a hybrid strong/weak coupling approach to jet quenching
}

\author{
Jorge Casalderrey-Solana ${ }^{\mathrm{a}}$, Doga Can Gulhan ${ }^{\mathrm{b}}$, José Guilherme Milhano ${ }^{\mathrm{c}, \mathrm{d}}$, Daniel \\ Pablos $^{\mathrm{a}}$, Krishna Rajagopal ${ }^{\mathrm{b}}$ \\ ${ }^{a}$ Departament d'Estructura i Constituents de la Matèria and Institut de Ciències del Cosmos (ICCUB), Universitat de Barcelona, Martí i \\ Franquès 1, 08028 Barcelona, Spain \\ ${ }^{b}$ Laboratory for Nuclear Science and Department of Physics, Massachusetts Institute of Technology, Cambridge, MA 02139, USA \\ ${ }^{c}$ CENTRA, Instituto Superior Técnico, Universidade de Lisboa, Av. Rovisco Pais, P-1049-001 Lisboa, Portugal \\ ${ }^{d}$ Physics Department, Theory Unit, CERN, CH-1211 Genève 23, Switzerland
}

\begin{abstract}
We explore a novel hybrid model containing both strong and weak coupling physics for high energy jets traversing a deconfined medium. This model is based on supplementing a perturbative DGLAP shower with strongly coupled energy loss rate. We embed this system into a realistic hydrodynamic evolution of hot QCD plasma. We confront our results with LHC data, obtaining good agreement for jet $R_{A A}$, dijet imbalance $A_{J}$ and fragmentation functions.
\end{abstract}

Keywords: jets, quenching, AdS/CFT

\section{Introduction}

We study the quenching of energetic jets traversing a strongly coupled plasma via a hybrid strong/weak coupling model based on treating physics processes occurring at different energy scales differently. Since very high energy jets are created at a scale $Q \gg \Lambda_{Q C D}$ their production spectrum is obtained perturbatively and is therefore under good theoretical control. Subsequent parton showering is described by DGLAP evolution which relaxes the initial virtuality down to the hadronization scale. In the presence of a medium, travelling partons also participate in soft momentum transfers with the plasma constituents of the order of the temperature $T \gtrsim \Lambda_{Q C D}$. Since this scale is not large, we assume non-perturbative effects to be important. The treatment of these is inspired by gauge/gravity duality insights.

The latter technique determines the energy loss of probes travelling a strongly coupled medium of other nonAbelian theories which have holographical dual descriptions as gravitational theories. However, a description of the hard splittings is still lacking within this context, which means that a holistic explanation of the phenomena is at the moment not possible. For this reason we resort to a phenomenological model. We embed this hybrid model into a hydrodynamic description of expanding hot QCD matter and analyse several jet observables which we compare to LHC data.

\section{A Hybrid Model}

We generate hard events with PYTHIA [1], and assume that for these high virtuality processes modifications of nuclear parton distribution functions are small. To avoid unconstrained assumptions regarding hadronization and color reconnection we stay at partonic level, since fully reconstructed jets are expected to have little sensitivity to hadronization effects. 

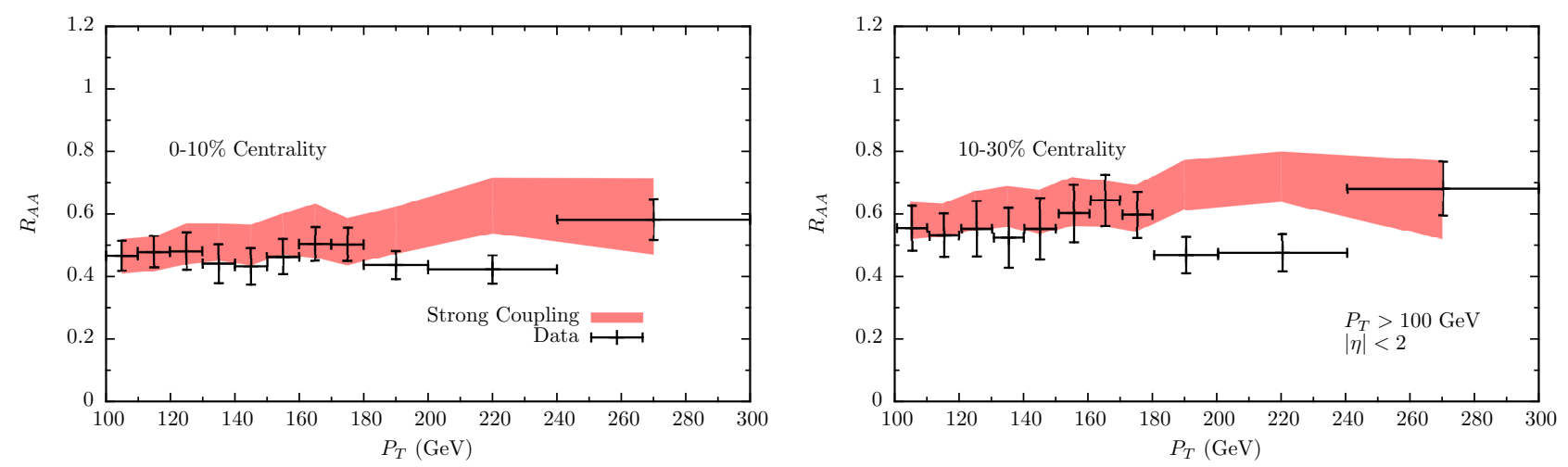

Figure 1. Jet $R_{A A}$ for two centralities. The only free parameter is fitted to the first bin of the left panel. Both the $p_{T}$ and centrality dependence are in agreement with the displayed data [3].

We use a formation time argument $\left(\tau_{f}=2 E / Q^{2}\right)$ to ascribe a space-time structure to the decay chain, for which the splitting probabilities remain as in vacuum. The system is embedded into a hydrodynamical model of expanding plasma with a realistic equation of state [2]. Since we are only interested in the strongly coupled phase, we stop subtracting energy from the jet when the local temperature of the plasma falls below $T_{C}$. This temperature is not unambiguously defined, so we explore the range $180<T_{C}<200 \mathrm{MeV}$ to gauge our theoretical uncertainties. We neglect energy loss in the hadronic phase.

Following [4] we explore an energy loss rate inspired by that of an energetic light quark traversing a slab of supersymmetric Yang-Mills plasma

$$
\frac{1}{E_{\text {in }}} \frac{d E}{d x}=-\frac{4 x^{2}}{\pi x_{\text {stop }}^{2} \sqrt{x_{\text {stop }}^{2}-x^{2}}}
$$

where $E_{\text {in }}$ is the initial energy of the excitation at its creation point and $x_{\text {stop }}$ the stopping distance. Keeping the energy and temperature dependence of the holographic calculation we parametrize this maximum distance by

$$
x_{\text {stop }}=\frac{1}{2 \alpha_{S C}} \frac{E_{\text {in }}^{1 / 3}}{T^{4 / 3}}
$$

where in the strong coupling calculation $\alpha_{S C}=1.05 \lambda^{1 / 6}$, with $\lambda$ the 't Hooft coupling. Since these calculations are not done in QCD itself we reinterpret $\alpha_{S C}$ as a dimensionless free parameter that we fit to data. Note that the path dependence in Eq. (1) is not simply $L^{2}$, but has a rather intricate dependence, with the presence of a Bragg-like peak.

This energy loss rate is determined for quarks. For adjoint degrees of freedom, gluons, it is known that the parametric dependence is the same as for quarks, but no exact formula has been yet derived. Since the gluons are represented by two strings, we prescribe that the stopping distance and loss rate for a gluon are the same as those for a quark with half the energy of the gluon. We interpret this factor of 2 as the ratio of the Casimirs of the two color representations in the large $N_{C}$ limit. This reasoning leads to

$$
x_{\text {stop }}^{G}=x_{\text {stop }}^{Q}\left(\frac{C_{F}}{C_{A}}\right)^{1 / 3}
$$

To bracket the sensitivity of the various jet observables we analyse to the microscopic dynamics, we explore other energy loss mechanisms inspired by perturbative calculations which contain different path length dependences. In particular, we implement an energy loss rate given by

$$
\frac{d E}{d x}=-\alpha_{R} \frac{C_{r}}{C_{F}} T^{3} x
$$

which captures the path length dependence of radiative energy loss (to logarithmic accuracy), the dominant mechanism at weak coupling. The $r$ in the Casimir stands for parton representation. We stress that this simplified implementation is not aimed at superseding other more sophisticated analyses of such processes. 

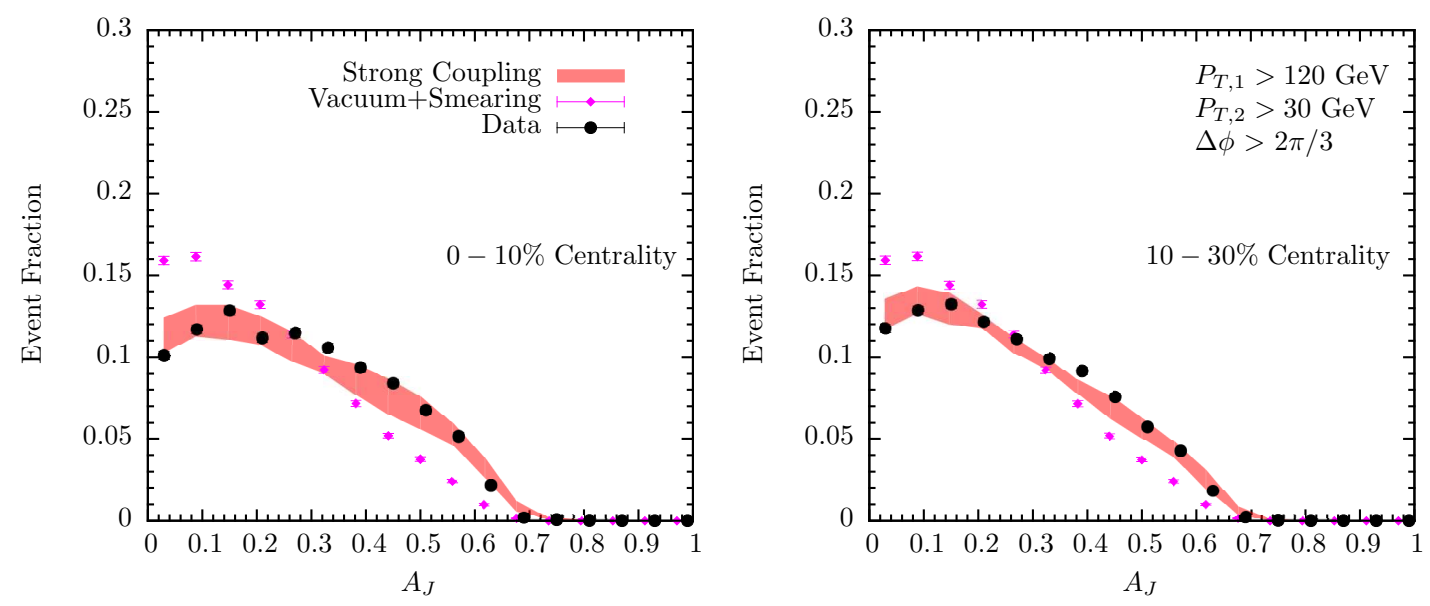

Figure 2. Dijet imbalance for two centralities. The strongly coupled bands follow experimental points satisfactorily. Data taken from [5] (systematic error not shown)

Finally, we also explore an energy loss rate with distinct path length dependence of the form

$$
\frac{d E}{d x}=-\alpha_{C} \frac{C_{r}}{C_{F}} T^{2}
$$

which is inspired by collisional energy loss. For both benchmark models $\alpha_{C}$ and $\alpha_{R}$ are dimensionless fitting parameters. The complete description of the whole setup can be found in our main paper [6].

\section{Comparison with Data}

Following the experimental procedure, we reconstruct jets using the FastJet [7] anti- $k_{T}$ algorithm with $R=0.3$ within a rapidity range $|y|<2$. We constrain our models by fitting the jet $R_{A A}$ for $100<p_{T}<110 \mathrm{GeV}$ in the $0-10 \%$ centrality class. In Figure 1 we show the jet $R_{A A}$ for the strongly coupled model compared to preliminary CMS data [3] for two different centrality classes. The shape is weakly dependent on $p_{T}$. The model predicts the main features of the centrality dependence although the more peripheral bins deviate mildly from data (not shown); this may be due in part to the absence of any quenching at all in the hadron gas phase, whose relative importance becomes greater with diminishing centrality.

In Figure 2 we show the model prediction for the dijet imbalance for the same centralities. Since current data $[5,8]$ are not fully unfolded from resolution effects, a direct comparison with our model results is not possible. The CMS collaboration has shown that a simple smearing procedure [9] takes into account the main systematics of such effects. After applying this procedure, the strongly coupled calculation is in very good agreement with the data. This success is not exclusive of this model.

A useful way of establishing the capability for the different models to simultaneously describe these two observables is scanning their parameter space as shown in the left panel in Figure 3. We plot the mean dijet imbalance as a function of jet suppression for central collisions for different choices of the fitting parameter, which describe a curve in parameter space. The theoretical uncertainties in $T_{C}$ transform these curves into bands. Current systematic uncertainties cannot discriminate between the different model implementations. However, the modest separation of the bands displayed in the figure provides a quantification of the level of precision in jet data needed to discriminate between these very different underlying dynamics.

In the right panel in Figure 3 we have computed the ratio between in medium and vacuum partonic fragmentation functions. The points labelled as data correspond to three characteristic points of the measured ratio of hadronic fragmentation functions: the two crossing points at one and the minimum. The inspection of this plot strongly disfavours the collisional model. Admittedly, a direct comparison of our partonic calculations with data is less constraining for this observable. However, in general we expect that hadronization softens the fragmentation functions shifting all 

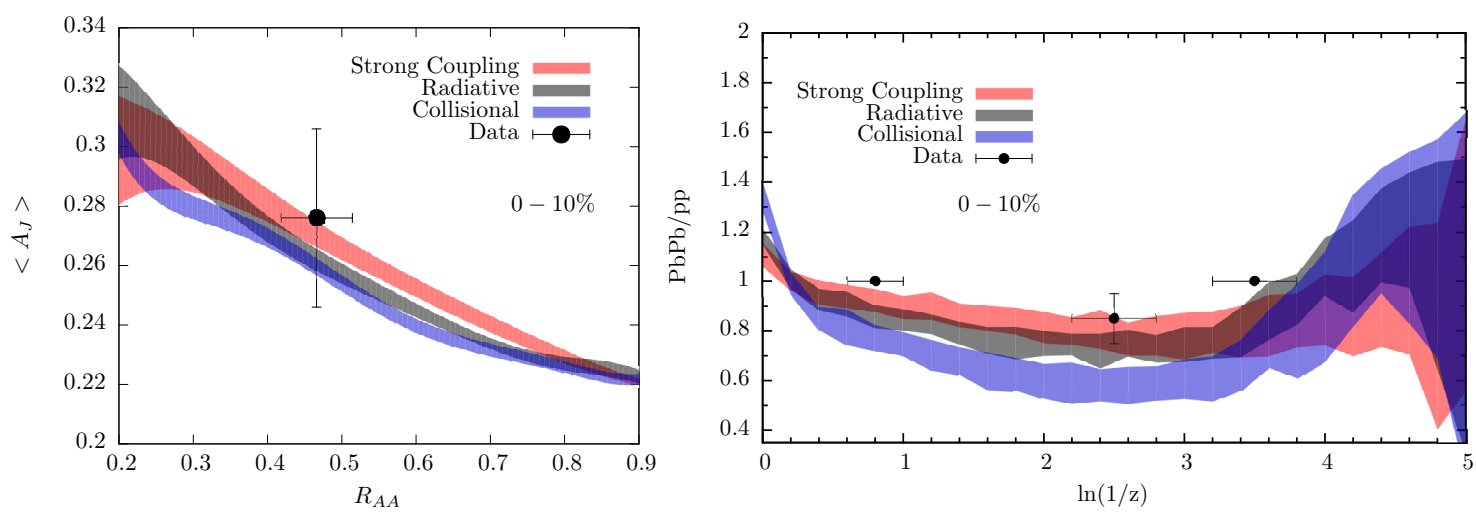

Figure 3. Left:Parameter space scan $\left\langle A_{J}\right\rangle$ vs $R_{A A}$ for central collisions. The chosen bin for $R_{A A}$ corresponds to the one used to fit the data Right: Ratio of partonic fragmentation functions for central collisions. The three data points shown correspond to the two crossings with unity and the location of the minimum of the hadronic fragmentation functions from CMS [10].

curves to the right, reinforcing our previous conclusion. While the strongly coupled model provides the best fit to data in hard and medium regions, it fails to reproduce the softest part. Nevertheless it is precisely this sector that is more sensitive to hadronization effects and to the possible enhancement of soft particles by the lost energy.

\section{Conclusions}

Despite the simplifications of the approach explored we observe a satisfactory agreement between our hybrid strongly coupled model and jet data both at the qualitative and quantitative level. The extracted values of the fitting parameter $\alpha_{S C}, 0.3<\alpha_{S C}<0.4$ are of order one, as expected from holographic calculations. Even though this success is encouraging, we notice that some of the observables considered have limited sensitivity to the underlying dynamics at present. Their sensitivity will improve as the systematic uncertainties in the data are reduced. It is also important to investigate further observables, such as photon+jet observables and heavy quark suppression.

\section{Acknowledgements}

JCS is a Ramón y Cajal fellow. JCS and DP are supported by the grants FP7-PEOPLE-2012- GIG-333786, FPA2013-40360-ERC, FPA2010-20807, 2009SGR502 and by the Consolider CPAN project. JGM is supported by Fundação para a Ciência e a Tecnologia (Portugal) under project CERN/FP/123596/2011 and contract 'Investigador FCT - Development Grant'. The work of DCG and KR was supported by the U.S. Department of Energy under cooperative research agreement DE-FG0205ER41360.

\section{References}

[1] T. Sjostrand, S. Mrenna, P. Z. Skands, JHEP 0605 (2006) 026. arXiv:hep-ph/0603175.

[2] T. Hirano, P. Huovinen, Y. Nara, Phys.Rev. C84 (2011) 011901. arXiv:1012.3955.

[3] Y. S. Lai for the CMS collaboration, these proceedings.

[4] P. M. Chesler, K. Rajagopal, Jet quenching in strongly coupled plasma arXiv:1402.6756.

[5] S. Chatrchyan, et al., Phys.Lett. B712 (2012) 176-197. arXiv:1202.5022.

[6] J. Casalderrey-Solana, D. C. Gulhan, J. G. Milhano, D. Pablos, K. Rajagopal, A Hybrid Strong/Weak Coupling Approach to Jet Quenching arXiv: 1405.3864

[7] M. Cacciari, G. P. Salam, G. Soyez, Eur.Phys.J. C72 (2012) 1896. arXiv:1111.6097.

[8] G. Aad, et al., Phys.Rev.Lett. 105 (2010) 252303. arXiv:1011.6182.

[9] Y. Yilmaz, Jet quenching in heavy-ion collisions at LHC with CMS detector, PhD thesis.

[10] M. Yaxian for the CMS collaboration, these proceedings. 